\title{
Studying User-defined iPad Gestures for Interaction in Multi-display Environment
}

\author{
Ekaterina Kurdyukova, Matthias Redlin, Elisabeth André \\ University of Augsburg \\ Universitaetsstrasse 6a, 56159 Augsburg, Germany \\ \{kurdyukova, redlin, andre\}@ informatik.uni-augsburg.de
}

\begin{abstract}
The paper investigates the iPad gestures that users naturally perform for data transfer. We examine the transfer between two iPads, iPad and a tabletop, and iPad and a public display. Three gesture modalities are investigated: multitouch gestures, performed using the iPad display, spatial gestures, performed by manipulating iPad in 3D space, and direct contact gestures, involving the physical contact of iPad and another device. We report on the user choices of modalities and gesture types, and derive critical aspects for the design of iPad gestures.
\end{abstract}

\section{Author Keywords}

User-defined gestures, tablet PC, multi-display interaction

\section{ACM Classification Keywords}

H.5.2. Information interfaces and presentation: User Interfaces - Interaction styles, evaluation/methodology, user-centred design.

\section{General Terms}

Design, Experimentation, Human Factors

\section{MOTIVATION}

As predicted by Mark Weiser back in 1991 [1], tablet-sized devices, such as iPad, gain an increasing importance in the modern world. Among their rich interaction possibilities, tablet devices support the convenient gestural interaction. Gestural interaction is known to be intuitive, quick, comfortable [2], as well as fun and engaging [3]. However, a great challenge remains the design of the gestures that replicate the way people interact naturally.

The goal of this work is to find the gestures that users naturally perform with iPad ${ }^{1}$ for data transfer. By means of an experiment we study the gestures in three settings: iPad and iPad, iPad and a tabletop, iPad and a public display. We analyze the rationales behind the user choice of the gestures, and derive the guidelines for the gesture design.

This is the author's version of the work. It is posted here for your personal use. Not for redistribution. The definitive Version of Record was published in:

IUI'12, February 14-17, 2012, Lisbon, Portugal.

Copyright 2012 ACM 978-1-4503-1048-2

\section{STUDIES ON USER-DEFINED GESTURES}

User-defined gestures have been widely studied in the domains of full body interaction [4], tabletop interaction [5, $6,7]$, interaction on vertical surfaces $[8,9]$, as well as mobile interaction [10].

The research on natural gestures has also been done in the domains of multi-device $[11,12]$ and multi-display $[13,14]$ environments. For example, Kray et al. [2] studied userdefined mobile gestures that people perform for connection tasks between mobile devices, public displays, and tabletops.

However, not much work has been done so far to study the gestures people naturally perform with tablet-sized devices. Rekimoto [14] presented pen-based technique for the data transfer between tablet-sized devices. To enable the transfer, the technique exploits the touch screen of the tablet device. However, apart from the touch screen, a modern tablet device possesses more interaction possibilities enabled e.g. by integrated accelerometers or cameras. Therefore, there is a need for further research on tablet gestures, to understand which gestures are appreciated and found appropriate by the users.

\section{MODALITIES OF IPAD GESTURES}

Thinking of possible gestures that can be performed on the touch surfaces or with mobile devices, we can distinguish three gesture types for iPad:

- Multi-touch gestures are performed on the iPad screen.

- Spatial gestures are performed by rotating, tilting, or panning the iPad body.

- Direct contact gestures imply physical contact between the iPad body and the body of another device. The direct contact gestures can be also performed from a distance, imitating the real physical contact. For instance, the iPad display can reproduce the entire content of the remote display, similarly to the technique by Boring et al. [15].

${ }^{1}$ Here and further on we reference only to iPad. However, the discussion can be applied to any tablet PC device possessing similar physical properties and sensory equipment as iPad. 


\section{STUDYING iPAD GESTURES}

In order to understand which gestures and which modalities are intuitively chosen by the users when performing the transfer tasks on multiple displays, we arranged an experiment.

The experiment involved two transfer tasks, sending and receiving, performed in three settings: iPad-iPad, iPadTabletop, iPad-Public Display. The users had to perform the transfer of a large and small object in every setting. The order of the settings was counterbalanced.

The experiment was structured into two parts. Part 1 investigated the gestures that people perform spontaneously for the transfer in either setting. Here, the participants were free to choose any modality. However, since such spontaneous gestures may be strongly motivated by the experiences with other devices, they might not reflect the real user preferences. Part 2 aimed to correct this effect by giving the participants a chance to explore all possible modalities. Here the participants were asked to complete the tasks in either modality, in either setting, and then to express their preferences.

The experiment was conducted as a within-group test, individually with every participant. After a short introduction, the moderator explained the basic interaction techniques on iPad, avoiding any demonstrations. Then the participants were asked to complete Part1, Part2, give their gesture preference, and explain the choice.

Important to mention, in Part 1 two distances were examined in iPad-Public Display setting: closer and farther from the display. Thus, we could imitate the real life settings: often it is not possible to reach the display.

All in all, 20 persons took part in the test, 14 males and 6 females, aged between 21 and 55 (average 28,8), occupied as a carpenter, a sports teacher, law students, and IT researchers. Ten participants had experiences in the gesturebased entertainment consoles, 13 possess touch-enabled mobile devices, six participants had experiences with the tablet devices; among them only two possess an iPad; ten participants have never worked with a tabletop.

\section{RESULTS: USER-DEFINED GESTURES}

Among the gestures performed in Part 1, 58\% included multi-touch gestures, $17 \%$ spatial gestures, and 25\% direct contact gestures.

\section{iPad-iPad Setting}

Multi-touch gestures for sending of the large and the small objects were performed by $76 \%$ of the participants by dragging the object with the finger from the participant's own iPad in the direction of the other iPad (see Fig.1, left). Receiving with multi-touch was performed by $50 \%$ of the persons, similarly: by dragging the object with the finger, either directly from the other iPad or only using the surface of the own iPad. The participants usually placed their iPad in the same plane with the source iPad, organizing a common surface for dragging.
Spatial gestures for sending were performed by $20 \%$ of the participants by pouring out of the object from the iPad so that the object "slides" to the other iPad (see Fig. 2, left). For receiving, however, spatial gestures were generally found inappropriate. Just two participants performed them by pulling the iPad from the source iPad in the direction of their body.

Direct contact gestures for sending were performed by $4 \%$ of the participants by placing or "posting" the object on the other iPad by holding the own iPad over the receiving iPad. For receiving about $50 \%$ of the participants placed the iPad over the other iPad to copy the object or attract it by imagined gravity (see Fig. 3, left).
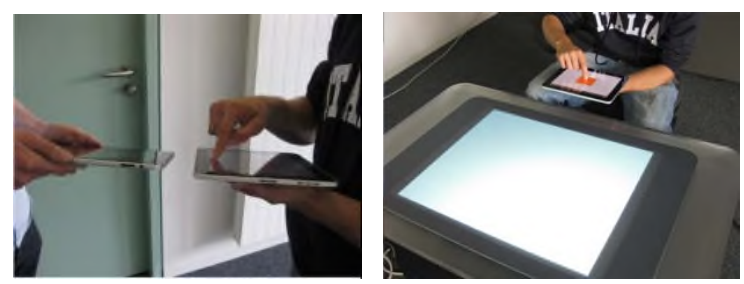

Figure 1. Sending the object with multi-touch gestures
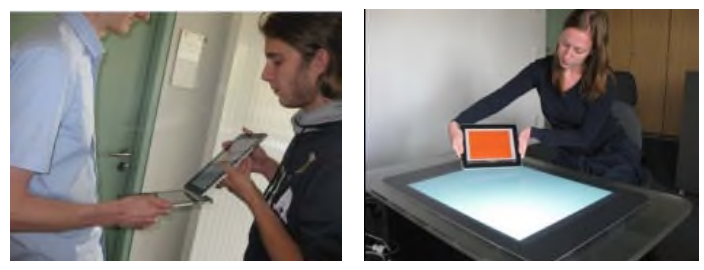

Figure 2. Sending the object with spatial gestures
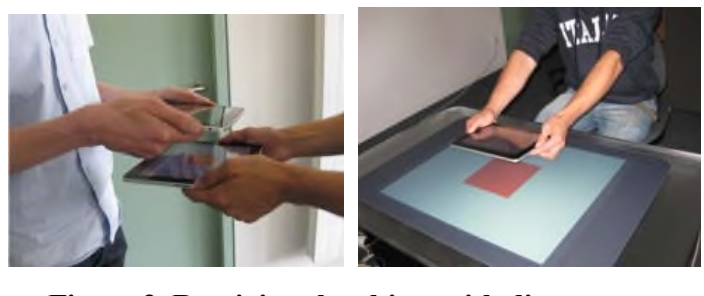

Figure 3. Receiving the object with direct contact
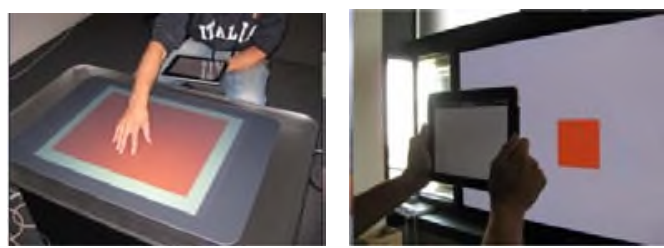

Figure 4. Receiving the object with multi-touch and direct contact gestures

\section{iPad-Tabletop Setting}

For sending the majority of the participants $(80 \%)$ performed multi-touch gesture: dragging the object with finger from iPad in the direction of the table (see Fig. 1, right). Just few participants touched the table. For receiving the majority (70\%) was dragging the object from the table to iPad (see Fig. 4, left). Most of participants touched both the table and iPad. 
Spatial gestures for sending were performed by $12 \%$ of the participants. They mostly included pouring the object out from iPad to the table (see Fig. 2, right). Some participants shook the device additionally, to "make sure the object slides down". For receiving almost no one has performed spatial gestures, commenting that it is hard to find a good match.

Direct contact gestures for sending were performed by $8 \%$ of the participants, by "posting" the object to the table almost placing the device physically on the surface. For receiving, about $30 \%$ of the participants performed holding the iPad over the table to "capture" the small object (see Fig. 3, right) or placing the iPad at the table to "imprint" the large object. Seven participants placed iPad on the table before the interaction.

In both iPad-iPad and iPad-Tabletop settings, for the transfer of the large objects the participants often used several fingers. The tasks involving the small object were performed mostly with one finger. Moreover, the large objects were frequently scaled down before the transfer.

\section{iPad - Public Display Setting}

For sending the majority of the participants $(75 \%)$ chose multi-touch modality by dragging the object with the finger from iPad in the direction of the display. For receiving, however, multi-touch gestures were found rather inappropriate. Just few participants performed pulling the corner of iPad by multiple dragging motions, reminding scratching movements, commenting however that the gesture is not really suitable for the transfer.

Spatial gestures for sending were performed by $15 \%$ of the participants. The gestures included intensive jerky "throwing" of the object in the horizontal plane or "throwing the object" by tilting iPad from the body to the horizontal position, orthogonal to the display. For receiving, $20 \%$ of the participants performed the gesture: „scooping up " the object from the large display.

Direct contact gestures for sending were performed by $10 \%$ of the persons by "posting " or ,pinning up " the object on the large display. For receiving, direct contact gestures were performed by the majority (75\%). To receive a small object the participants were ,picking up " or "capturing" the small object from the display (see Fig. 4, right); to receive a large object they touched an imagined marker at the large display to copy the content.

Difference distances influenced only the sending gestures. The further the participant stayed from the display, the less multi-touch and the more spatial gestures were performed. The phenomenon can be explained by the perceived weight of the transferred object. Throwing with the finger gives the object a minor acceleration. Thus, it can "fly" only over a short distance. Throwing using the whole iPad body can produce a stronger acceleration. Therefore, the object can securely "fly" over a longer distance and not to "get lost" on the way.
Part 2 gave the inexperienced participants the chance to try all possible gesture modalities, and perhaps correct their preferences. Indeed, in Part 2 the participants expressed more preferences for spatial and direct contact gestures, in iPad-iPad and iPad-Tabletop settings. These modalities were perceived more creative and more enjoyable than multi-touch gestures. Especially, the spatial gestures were referred as "more funny to perform". However, in iPad$i P a d$ setting, even well-matching spatial gestures were often perceived as unreliable. In iPad-Tabletop setting, this disadvantage was diminished due to the presence of the table. Placing iPad on a solid surface, people felt they can better control the transfer.

Direct contact modality was less popular in iPad-Tabletop setting compared to Part1. The physical dimensions of iPad and the table were reported to be too different to find a well-matching metaphor for a direct transfer (in other words to "pack" the object directly to iPad).

To summarize, the participants favoured the multi-touch modality almost in all the settings. The exception were only the receiving gestures in $i P a d-P u b l i c$ Display setting which was most frequently performed with direct contact.

\section{DESIGNING IPAD GESTURES}

Below we emphasize the aspects important to consider when designing iPad gestures.

\section{Physical Shape}

The flat page-like shape of iPad device affects its physical disposition to the other devices, its orientation, and the set of possible spatial gestures.

The physical touch of other devices occurred mostly in iPad-iPad setting and especially in iPad-Tabletop setting. The flat shape of the iPad body invites to place it on a flat horizontal surface.

The flat shape also restricts the spatial gestures that can be performed with iPad. Thus, the usage of spatial rotations is rather provoked by the distance to the target device. The further the device, the more spatial gestures are performed. Therefore, spatial gestures were popular in $i P a d-i P a d$ and iPad-Public Display settings.

Physical shape also influences the iPad orientation: during the transfer iPad is often oriented in the same plane with the display. For example, it is placed boarder to boarder to the other iPad or a tabletop arranging a continuous surface.

\section{Thinking of Flat Metaphors}

Both experienced and inexperienced users rely on real-life metaphors when thinking of well-matching gestures. The most common iPad metaphor refers to a tray (or a salver). The object on the iPad screen is associated with a flat physical object lying on the tray. The iPad is usually held with two hands; its display is associated with the top side of the tray. Therefore, iPad is almost never turned around. If iPad is manipulated in 3D space, the final position lies always in the horizontal plane, oriented by the "top of the 
tray". Grabbing the objects from the other surfaces is performed similarly to the collection of pieces to the tray.

The object on the tray is perceived as a physical one, having its weight and size. Therefore, the larger the object, the more effort the user needs to move it; the more power needs to be applied to throw the object away from the tray. The gestures for the larger objects require stronger acceleration and involve more fingers.

The metaphor of a flat tray is often transformed into the plate metaphor. The participants often performed pouring out gesture reminding pouring out of a liquid from a plate. Here again the size of the object was mapped onto its weight. The plates with the "heavier" objects were additionally shaken, to ensure the object slides down from the plate.

The metaphor of a spade or a shovel was often applied to receiving tasks. The participants tried to scoop up the object from the source display using iPad as a shovel. Interestingly, the metaphor was applied to both horizontal and vertical surfaces.

\section{Privacy Matters}

An important aspect in the design of iPad gestures concerns the private space. The gestures involving the touch of another private device (iPad) are seen as an intrusion into the person's private space. This concerns both the finger interaction and the direct contact of the device body. Even the contactless interaction which presumes a close proximity of two devices (see e.g. Fig. 1, left) was seen slightly privacy critical.

Another concern relates to the direct contact gestures as the demonstration of the interaction on public. Direct contact was criticized by potentially drawing public attention and by the impossibility to interact unnoticed from a distance.

\section{CONCLUSION}

The paper presented a study on iPad gestures that are naturally chosen for the data transfer in multi-display settings. The results show that the choice of the gestures is strongly influenced by the physical shape of iPad, the associated metaphors, and the privacy concerns of the users.

\section{ACKNOWLEDGMENTS}

This research is partly sponsored by OC-Trust project (FOR 1085) of the German research foundation (DFG).

\section{REFERENCES}

1. Mark Weiser. The computer for the 21st century. Scientific American Special Issue on Communications, Computers, and Networks, September, 1991.

2. Kray, C., Nesbitt, D., Dawson, J., and Rohs, M. Userdefined gestures for connecting mobile phones, public displays, and tabletops. In Proc. MobileHCI 2010.

ACM Press (2011), 239-248.

3. Bianchi-Berthouze, N., Kim, W. W., and Patel, D. Does Body Movement Engage You More in Digital Game Play? and Why? In Proc. ACII 2007, Springer-Verlag (2007), 102-113.

4. Shotton, J., Fizgibbon, A., Cook, M., Sharp, T., Finocchio, M., Moore, R., Kipman, A., and Blake, A. Real-time human pose recognition in parts from single depth images. In IEEE Computer Vision and Pattern Recognition (CVPR) 2011, IEEE.

5. Wobbrock, J.O., Morris, M.R., and Wilson, A.D. Userdefined gestures for surface computing. In Proc. CHI 2009. ACM Press (2009), 1083-1092.

6. Rekimoto, J. SmartSkin: An Infrastructure for Freehand Manipulation on Interactive Surfaces. In Proc. CHI 2002, ACM Press (2002), 113-120.

7. Wu, M. and Balakrishnan, R. Multi-Finger and Whole Hand Gestural Interaction Techniques for Multi-User Tabletop Displays. In Proc. UIST 2003, ACM Press (2003), 193-202.

8. Baudisch, P., Cutrell, E., Robbins, D., Czerwinski, M., Tandler, P., Bederson, B., and Zierlinger, A. Drag-andpop and drag-and-pick: Techniques for accessing remote screen content on touch- and pen-operated systems. In Proc. INTERACT 2003, Springer Verlag (2003), 57-64.

9. Bezerianos, A. and Balakrishnan, R. The Vacuum: Facilitating the Manipulation of Distant Objects. In Proc. CHI 2005, ACM Press (2005), 361-370.

10. Ruiz, J., Li, Y., and Lank, E. User-defined motion gestures for mobile interaction. In Proc. CHI 2011, ACM Press (2011), 197-206.

11.Lee, H., Jeong, H., Lee, J., Yeom, K., and Park, J. Gesture-Based Interface for Connection and Control of Multi-device in a Tabletop Display Environment, In Proc. HCI 2009, Springer Verlag (2009), 216-225.

12. Yatani, K., Tamura, K., Hiroki, K., Sugimoto, M., and Hashizume, H. Toss-it: intuitive information transfer techniques for mobile devices. In Proc. CHI 2005 Extended Abstracts. ACM Press (2005), 1881-1884.

13. Lee, J., Lee, J., Kim, H., and Kim, J. Gesture-Based Interactions on Multiple Large Displays with a Tabletop Interface, In Proc. UAHCI 2007, Springer Verlag (2007), 936-942.

14. Rekimoto, J. Pick-and-Drop: A Direct Manipulation Technique for Multiple Computer Environments, In Proc. UIST 1997, ACM Press (1997), 31-39.

15. Boring, S., Baur, D., Butz, A., Gustafson, S., and Baudisch, P. Touch projector: mobile interaction through video. In Proc. CHI 2010. ACM Press (2010), 2287-2296. 